\title{
Improved Biocompatibility of Intra-Arterial Poly-L-Lactic Acid Stent by Tantalum Ion Implantation : 3-Month Results in a Swine Model
}

\author{
Kangmin Kim, ${ }^{1, *}$ Suhyung Park, ${ }^{2, *}$ Jeong Hwan Park, ${ }^{3}$ Won-Sang Cho, ${ }^{1}$ Hyoun-Ee Kim, ${ }^{2}$ Sung-Mi Lee, ${ }^{2}$ Jeong Eun Kim, \\ Hyun-Seung Kang, ${ }^{1}$ Tae-Sik Jang ${ }^{4}$ \\ Department of Neurosurgery,' Seoul National University Hospital, Seoul National University College of Medicine, Seoul, Korea \\ Department of Materials Science and Engineering, ${ }^{2}$ Seoul National University, Seoul, Korea \\ Department of Pathology, ${ }^{3}$ Seoul Metropolitan Government - Seoul National University Boramae Medical Center, Seoul, Korea \\ Department of Materials Science and Engineering, ${ }^{4}$ Chosun University, Gwangju, Korea
}

Objective : Biodegradable poly-L-lactic acid (PLLA) with a highly biocompatible surface via tantalum (Ta) ion implantation can be an innovative solution for the problems associated with current biodegradable stents. The purpose of this study is to develop a Taimplanted PLLA stent for clinical use and to investigate its biological performance capabilities.

Methods: A series of in vitro and in vivo tests were used to assess the biological performance of bare and Ta-implanted PLLA stents. The re-endothelialization ability and thrombogenicity were examined through in vitro endothelial cell and platelet adhesion tests. An in vivo swine model was used to evaluate the effects of Ta ion implantation on subacute restenosis and thrombosis. Angiographic and histologic evaluations were conducted at one, two and three months post-treatment.

Results : The Ta-implanted PLLA stent was successfully fabricated, exhibiting a smooth surface morphology and modified layer integration. After Ta ion implantation, the surface properties were more favorable for rapid endothelialization and for less platelet attachment compared to the bare PLLA stent. In an in vivo animal test, follow-up angiography showed no evidence of in-stent stenosis in either group. In a microscopic histologic examination, luminal thrombus formation was significantly suppressed in the Ta-implanted PLLA stent group according to the 2-month follow-up assessment (21.2\% vs. 63.9\%, $p=0.005)$. Cells positive for CD 68 , a marker for the monocyte lineage, were less frequently identified around the Ta-implanted PLLA stent in the 1-month follow-up assessments.

Conclusion : The use of a Ta-implanted PLLA stent appears to promote re-endothelialization and anti-thrombogenicity.

Key Words : Absorbable implants · Lactic acid · Stents · Tantalum · Pig.

- Received : January 12, 2021 •Revised : March 9, $2021 ・$ Accepted : March 19, 2021

- Address for reprints : Hyun-Seung Kang

Department of Neurosurgery, Seoul National University Hospital, Seoul National University College of Medicine, 101 Daehak-ro, Jongno-gu, Seoul 03080, Korea Tel : +82-2-2072-2850, Fax : +82-2-744-8459, E-mail : kanghs@snuh.org, ORCID : https://orcid.org/0000-0002-6957-1907

\section{Tae-Sik Jang}

Department of Materials Science and Engineering, Chosun University, 309-3 Pilmun-daero, Dong-gu, Gwangju 61452, Korea

Tel : +82-62-230-7199, Fax : +82-62-608-5335, E-mail : tsjang@chosun.ac.kr, ORCID : https://orcid.org/0000-0003-3540-0525

*These authors contributed equally to this work and co-first authors.

This is an Open Access article distributed under the terms of the Creative Commons Attribution Non-Commercial License (http://creativecommons.org/licenses/by-nc/4.0) which permits unrestricted non-commercial use, distribution, and reproduction in any medium, provided the original work is properly cited. 


\section{INTRODUCTION}

Intra-arterial stenting is one of the most important treatment options for cerebral aneurysms. Although the use of bare metal stents in complex aneurysms has rapidly increased due to their superior mechanical strength and supportability, they remain permanently in the treated vessel and act as a foreign body, which may cause severe complications such as thrombus formation and arterial restenosis ${ }^{8)}$. A biodegradable stent that dissolves naturally within the blood vessel after implantation can be a potential solution for these problems, and many studies have been conducted to develop clinically relevant biodegradable stents, especially in the cardiovascular field $^{7,24)}$.

Poly-L-lactic acid (PLLA) is a widely utilized biodegradable polymer in biomedical applications because it can hydrolyze to a natural byproduct under physiological conditions ${ }^{24)}$. With regard to stents and the use of PLLA, however, several critical issues must be addressed ${ }^{6,16)}$. In particular, its strong hydrophobicity and innate hypersensitivity with an inflammatory response are regarded as the major causes of poor re-endothelialization and thrombosis ${ }^{19)}$. We have initiated studies to overcome these problems via plasma-based surface modification techniques, especially Tantalum (Ta) ion implantation. During this surface treatment, biocompatible Ta is directly implanted within the topmost surfaces of PLLA struts with a complex 3D geometry, forming a moderate hydrophilic surface and eliciting better cell-material interactions ${ }^{10,14)}$. In this study, we report for the first time a novel, biodegradable PLLA stent. This is realized by Ta ion implantation onto a laser-cut PLLA stent for intravascular implantation. The biological ef- fectiveness of the Ta ion implantation treatment on re-endothelialization and anti-thrombogenicity was evaluated in in vitro and in vivo model systems.

\section{MATERIALS AND METHODS}

The animal experiment conducted here was approved by the Institutional Animal Care and Use Committee of OSONG Medical Innovation Foundation (KBIO-IACUC-2018-015) and followed the "the Guiding Principles in the Care and Use of Animals" approved by the American Physiological Society.

\section{Stent preparation}

Fig. 1 shows a schematic illustration of the Ta implantation treatment of a PLLA stent. PLLA tubes (Poly[L-lactide], 38; 3 $\mathrm{mm}$ outer diameter; $2.75 \mathrm{~mm}$ inner diameter; length, $10 \mathrm{~mm}$ ) were purchased from Biogeneral, Inc. (San Diego, CA, USA). Femtosecond laser processing (Spitfire Ace; Spectra Physics, Andover, MA, USA) was utilized to manufacture a PLLA polymer stent under optimized conditions of the pulse energy, scan speed, and spot size. Before the surface treatment, the stent samples provided were ultra-sonicated in ethanol for 10 minutes. Using a DC magnetron sputtering device (Ultech Co. Ltd., Daegu, Korea), Ta was implanted into the stent surface. The chamber was maintained in a vacuum state up to $5 \times$ $10^{-4} \mathrm{~Pa}$ for 90 minutes and argon gas was then flowed until the inner pressure reached $0.6 \mathrm{~Pa}$. Three different parts of the stent were marked at constant circumferential intervals for a uniform surface treatment. The three sections were attached to the substrate sequentially and sputtering onto the section

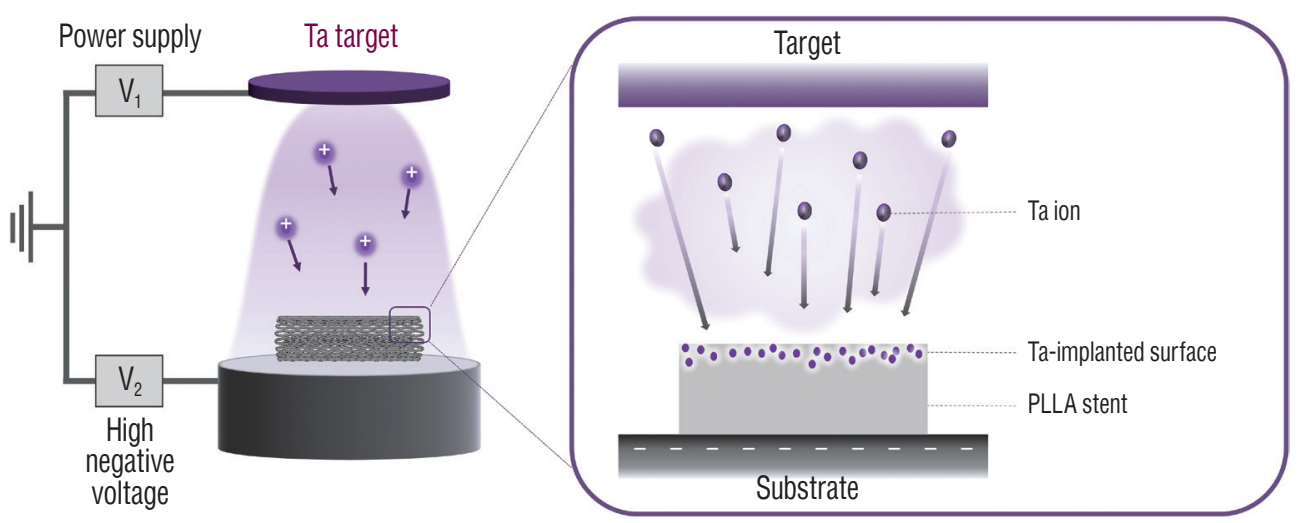

Fig. 1. Schematic illustration of the tantalum (Ta) implantation treatment on a poly-L-lactic acid (PLLA) stent. Ta ions were accelerated toward the surface of the PLLA stent, where high negative voltage was applied, forming a Ta-implanted polymer layer. 
was conducted for 20 seconds in each case. High negative voltage of $2000 \mathrm{~V}$ was applied to the substrate, and the Ta target power was $12 \mathrm{~W}$.

For an in vivo animal experiment, a radio-opaque marker was adopted at the end of the stent using gold wire $(0.1 \mathrm{~mm}$ thickness; Alfa Aesar, Ward hill, MA, USA). The PLLA stent was then mounted onto a $4 \times 20 \mathrm{~mm}$ non-compliant coronary balloon (Genoss, Suwon, Korea) by means of a crimping process (Fig. 2). Various sizes of balloons (3-5 mm) and different pressure levels (8-12 atm) were tested in order to deploy the stent successfully while maintaining its stable structure. The balloon size ( $4 \mathrm{~mm}$ ) and pressure (10 atm) used in this study were selected as a result. The arterial coverage ratio by the stent was $27.3 \%$. The control samples were a bare PLLA stent without a surface treatment.

\section{In vitro test}

The stents were observed via optical microscopy to confirm the maintenance of the structure after the surface treatment. The surface morphology and roughness $(\mathrm{Ra})$ of the bare and Ta-implanted PLLA stents were examined using a scanning electron microscope (SEM) and a confocal laser microscope,

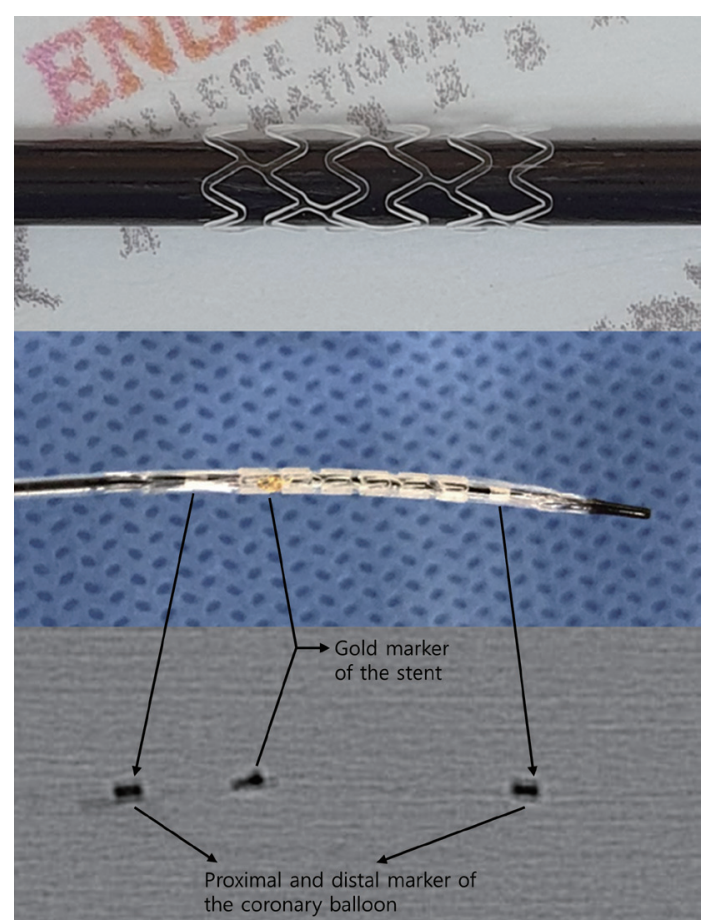

Fig. 2. Poly-L-lactic acid (PLLA) stent manufactured by laser cutting. The PLLA stent is mounted on a coronary balloon with a gold marker, which is identified in the fluoroscopic image. respectively. The wettability of the surface was evaluated by dropping 3.0 $\mu \mathrm{L}$ of distilled water onto it and fitting the droplet in a circle using a water contact angle (WCA) analyzer. For a WCA test of the luminal surface of a Ta-implanted sample, a PLLA tube, only vertical half of which had undergone a laser cutting process, was treated via Ta implantation to mimic a luminal side of a Ta-implanted stent. Ta-implanted PLLA stents were fabricated as transmission electron microscope (TEM) specimens using a focused ion beam after carbon deposition to evaluate the content and depth of the implanted $\mathrm{Ta}$ on the surface with an energy-dispersive spectroscopy line scan analysis by TEM. For a mechanical test, a flat plate test was conducted by referring to an earlier method ${ }^{12)}$. The stent was placed between two parallel plates and compression force was applied until the gap distance reached $50 \%$ of the diameter of the stent. Human umbilical vein endothelial cells (HUVECs; CRL-1730), purchased from American Type Culture Collection, were utilized to evaluate the endothelialization capabilities. All samples were sonicated in $70 \%$ ethanol for 10 minutes and then exposed to ultraviolet light for 1 hour. The cells were seeded at a density of $5 \times 10^{-4}$ cells $/ \mathrm{mL}$ in an endothelial cell basal medium-2 (Lonza, Walkersville, MD, USA) with $10 \%$ fetal bovine serum and $1 \%$ penicillin-streptomycin. They were cultured in a humidified incubator containing $5 \% \mathrm{CO}_{2}$ at $37^{\circ} \mathrm{C}$ and obtained after 1,4 , and 7 days. To visualize the spreading morphology and area of the cells on each specimen through confocal laser scanning microscopy (CLSM), they were treated with $4 \%$ paraformaldehyde (Sigma-Aldrich, St. Louis, MO, USA), 0.1\% Triton X-100 (Sigma-Aldrich) and 1\% bovine serum albumin (Sigma-Aldrich) in phosphate-buffered saline and then stained by phalloidin (Invitrogen, Waltham, MA, USA) and 4, 6-diamidino-2-phenylindole (Invitrogen) for the cytoplasm and nuclei, respectively. As a proliferation test, the seeding density was $3 \times 10^{4}$ cells $/ \mathrm{mL}$ and the absorbance at $492 \mathrm{~nm}$ was measured according to a methoxyphenyl tetrazolium salt assay with 3-(4,5-dimethylthiazol2-yl)-5-(3-carboxymethoxyphenyl)-2-(4-sulfophenyl)-2H-tetrazolium.

An in vitro platelet adhesion test was conducted as the sterilized stents were incubated in diluted platelet-rich plasma (PRP) for 1 hour. After washing out the remaining platelets, the numbers of platelets adhering to each stent were quantified using a lactate dehydrogenase assay by Multiskan GO (Thermo, Vantaa, Finland). For a SEM analysis, the samples 
were placed into contact with PRP at a higher concentration for one hour, washed, fixed with 2.5\% glutaraldehyde, and

(A)
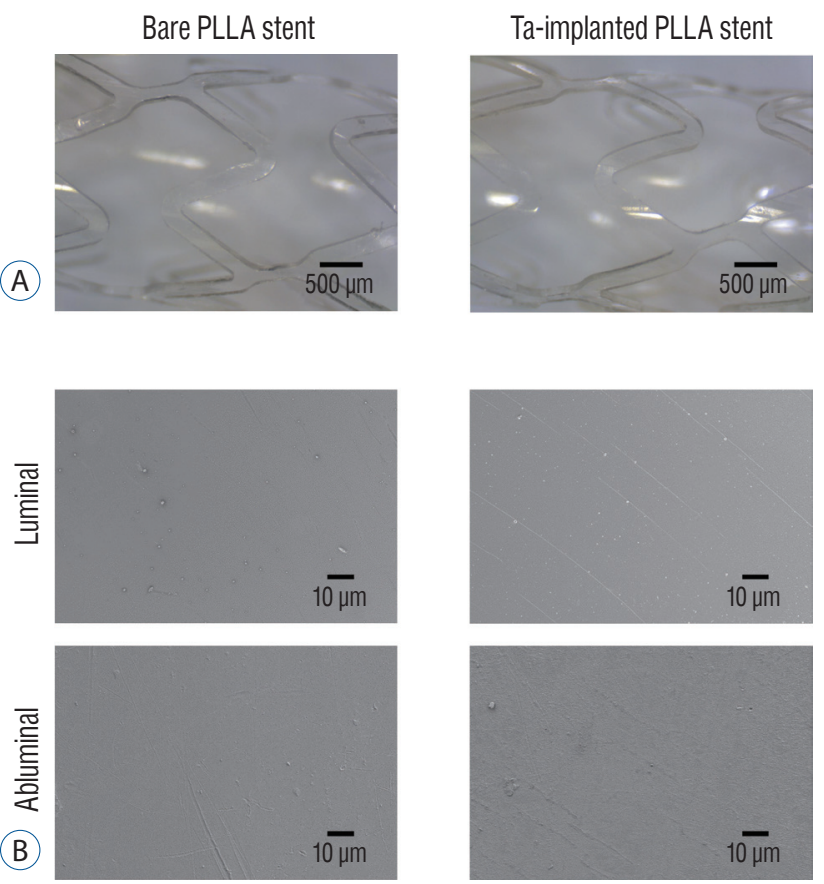

Fig. 3. Surface morphology of the stent. Optical (A) and scanning electron microscope (B) images of bare and tantalum (Ta)-implanted poly-L-lactic acid (PLLA) stents. The PLLA stent shows a smooth surface. There is no difference in the surface morphology of the PLLA stent before and after Ta implantation treatment. then dehydrated.

\section{In vivo test}

In total, four specific-pathogen-free male swine of about 30 $\mathrm{kg}$ were used for the in vivo test. Antiplatelet drugs (aspirin $100 \mathrm{mg}$ and clopidogrel $75 \mathrm{mg}$ daily) were administered 1 week before stent placement and were maintained until the animals were sacrificed.

For stent deployment, a 6 Fr femoral sheath was inserted, and a 6 Fr guiding catheter was positioned in the common carotid artery using a 0.035 -inch guide wire under angiographic

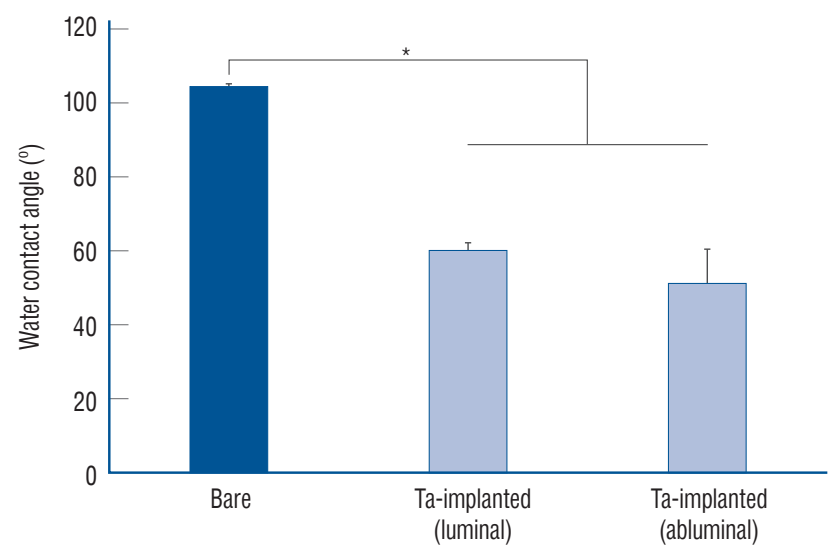

Fig. 5. Water contact angle test. The tantalum $(\mathrm{Ta})$ treatment increased the wettability of the stent. ${ }^{*} p<0.05$.
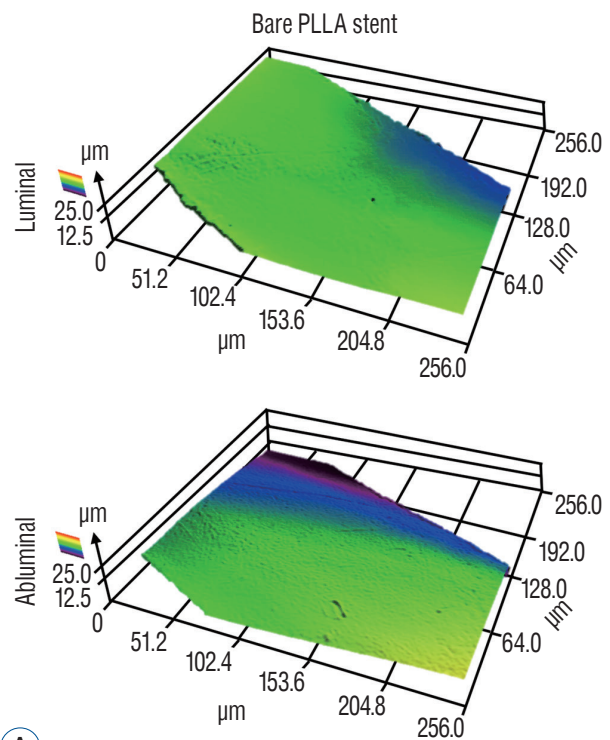

(A)

Fig. 4. Roughness of the stent. A : Surface topography 3D maps of bare a
significant difference in the calculated average roughness on each surface.
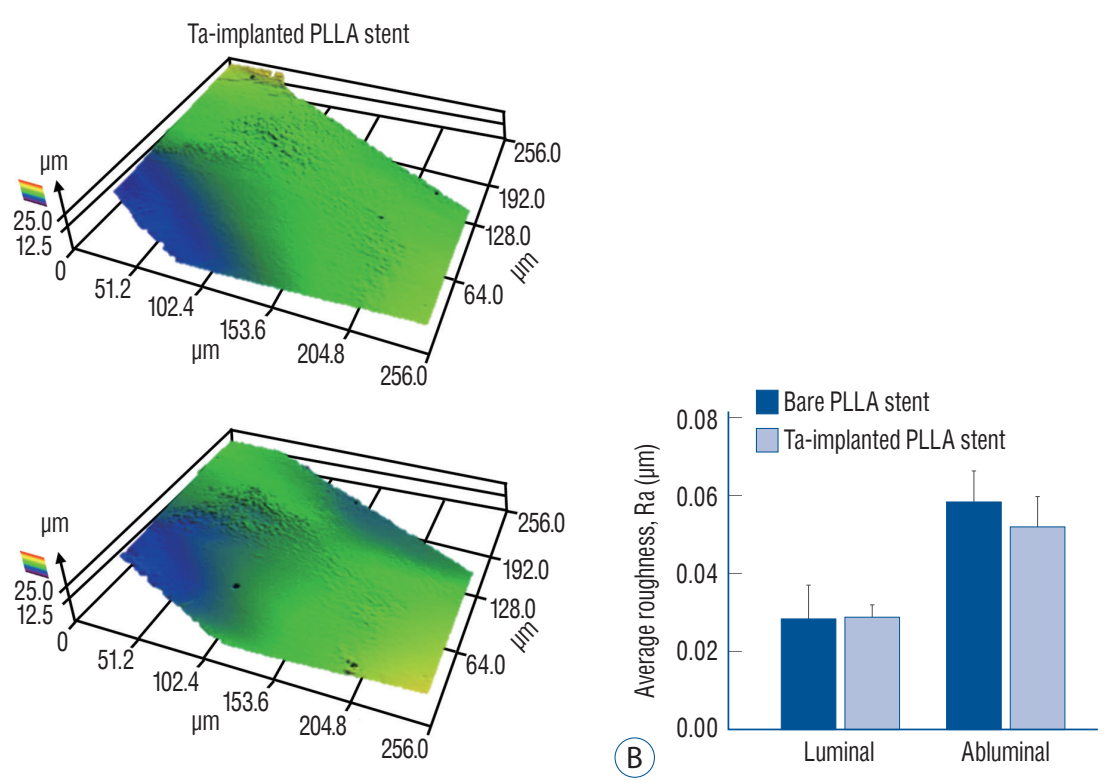
guidance (Artis zee, Siemens, Germany). Balloon-mounted PLLA stents were deployed in the external carotid artery (ECA) with diameters of 2.6 to $3.2 \mathrm{~mm}$. Two Ta-implanted PLLA stents were deployed in the right ECA and two bare PLLA stents were placed in the left ECA. Ultimately, sixteen PLLA stents were successfully deployed in the four swine. All procedures were performed under general anesthesia by a certified veterinarian. Anesthesia was induced with an intramuscular injection of Zoletil ${ }^{\circledR}$ (Virbac, Carros, France) $4 \mathrm{mg} / \mathrm{kg}$ and Rompun ${ }^{\circledR}$ (Bayer, Leverkusen, Germany) $2.2 \mathrm{mg} / \mathrm{kg}$. It was maintained using an inhaled agent (sevoflurane) and oxy-

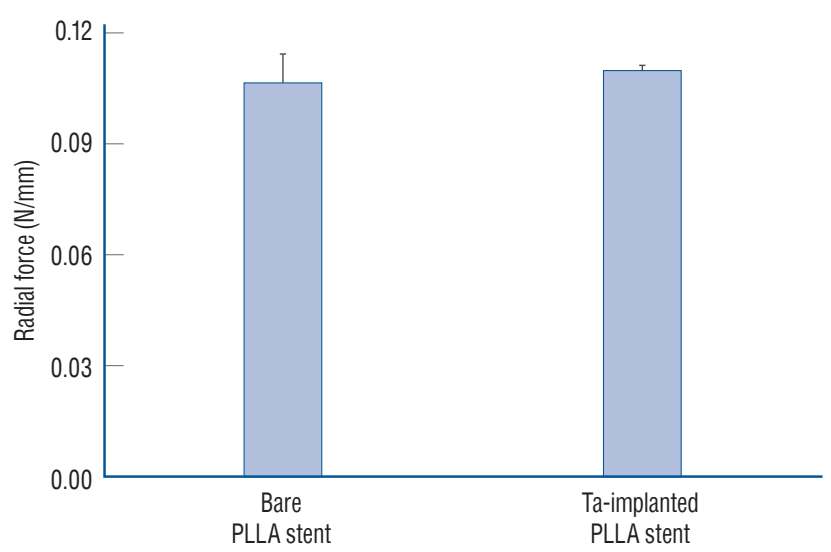

Fig. 6. Radial force of the poly-L-lactic acid (PLLA) stent (no significant difference).

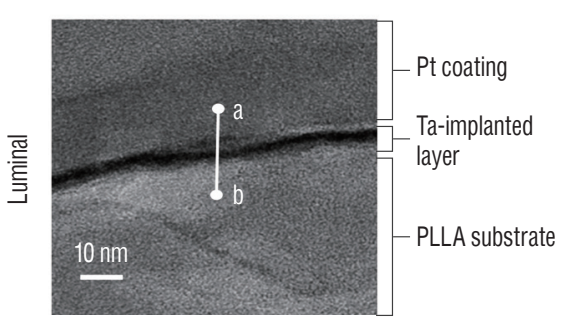

(B)

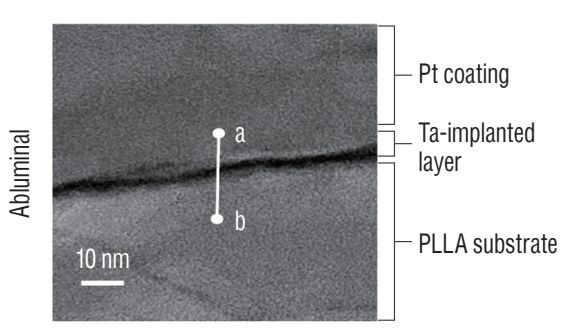

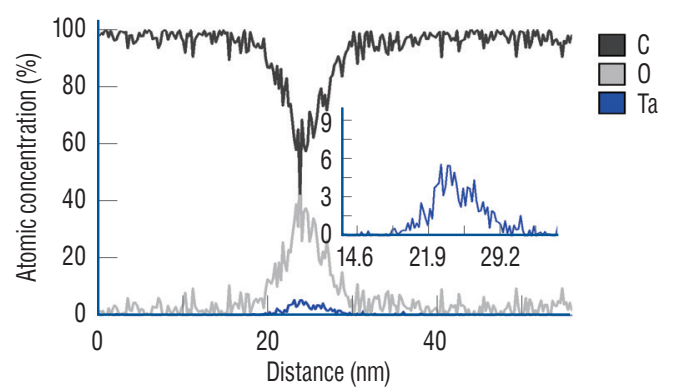

gen during the procedures.

Animals were sacrificed at $1(\mathrm{n}=2), 2(\mathrm{n}=1)$, and $3(\mathrm{n}=1)$ months after stent deployment. A follow-up angiography was performed to confirm arterial patency before sacrifice. One Ta-implanted stent at 1-month follow-up was lost during the harvesting procedure and thus 15 stents were harvested. Three paraffin blocks (the proximal, middle, and distal parts) and one resin block were made from each stent sample. For a histologic analysis, hematoxylin and eosin) staining and immunohistochemical staining for CD 31, CD 34, CD 68, and antismooth muscle cell alpha actin were performed. The histologic analysis included luminal thrombus $(\%=100 \times$ [luminal thrombus area/luminal area]), neointimal hyperplasia and stenosis, as suggested by Schwartz et al. ${ }^{18)}$, and inflammation and fibrosis according to a quantitative method to evaluate local biological effects after the implantation of the prosthesis in this case ${ }^{9,15}$. All histologic analyses were performed by an independent, blinded and certified pathologist. SPSS software (version 20; IBM corp., Armonk, NY, USA) and MedCalc for Windows (version 19; MedCalc Software, Ostend, Belgium) were used for the statistical analysis. A value of $p<0.05$ was considered statistically significant.

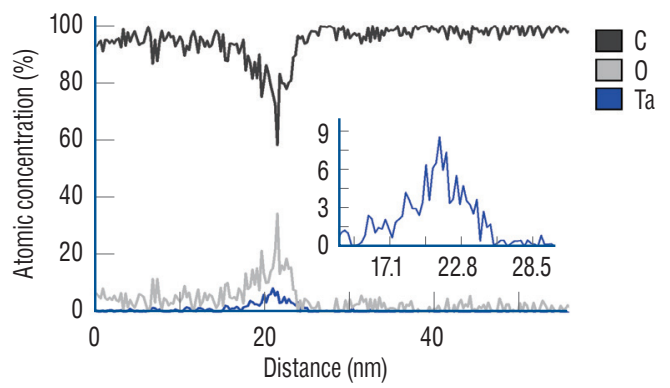

Fig. 7. Tantalum layer of the poly-L-lactic acid (PLLA) stent. Representative cross-sectional transmission electron microscope (TEM) image and the TEM/ energy-dispersive spectroscopy line profile with $\mathrm{C}, \mathrm{O}$, and tantalum $(\mathrm{Ta})$ along the white line from points a to $\mathrm{b}$ on the (A) luminal surface and (B) the abluminal surface of the Ta-implanted PLLA stent. 


\section{RESULTS}

\section{Surface characterization}

After the Ta ion implantation treatment, the PLLA stent had an opaque and colorless appearance, and its complex shape
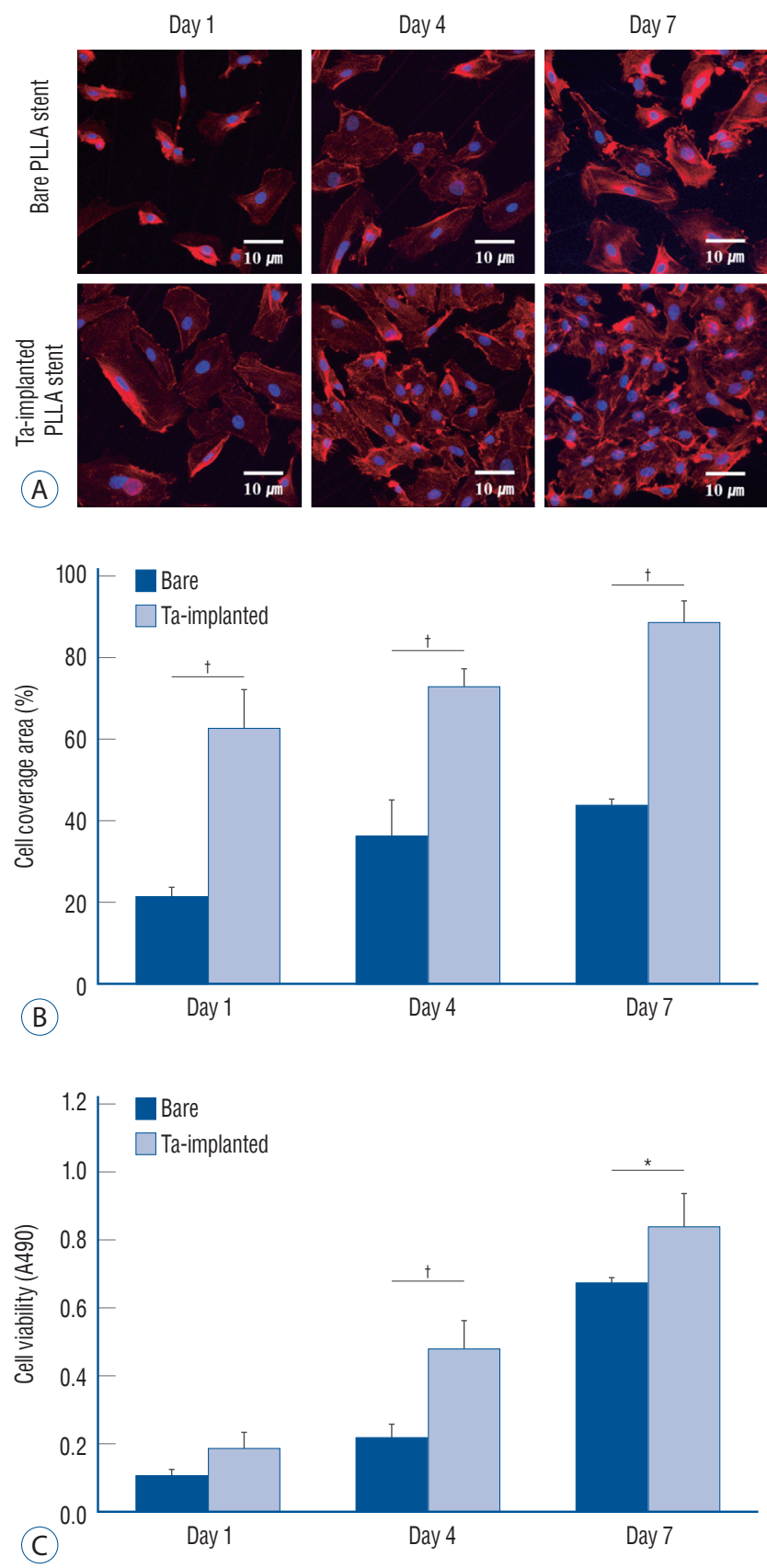

Fig. 8. Endothelialization test. A : Confocal laser scanning microscopy images of adhered endothelial cells. Surface coverage of endothelial cells (B) and cell viability (C) on bare and tantalum (Ta)-implanted poly-Llactic acid (PLLA) surfaces after 1, 4, and 7 days of culturing, respectively. ${ }^{*} p<0.05 .{ }^{\dagger} p<0.01$. was well maintained without any deformation or distortion (Fig. 3). Despite the several manufactured tracks on the stent surfaces before and after the surface treatment, their average surface Ra values were only approximately $0.028 \pm 0.008 \mu \mathrm{m}$ and $0.028 \pm 0.003 \mu \mathrm{m}$ for the bare and Ta-implanted PLLA stents, respectively, representing excellent micro-scale flatness on both surfaces (Fig. 4). As shown in Fig. 5, the WCAs (o) of the luminal and abluminal sides of the Ta-implanted samples were $60.0^{\circ} \pm 1.88^{\circ}$ and $51.1^{\circ} \pm 9.12^{\circ}$, respectively, while it was

(A)
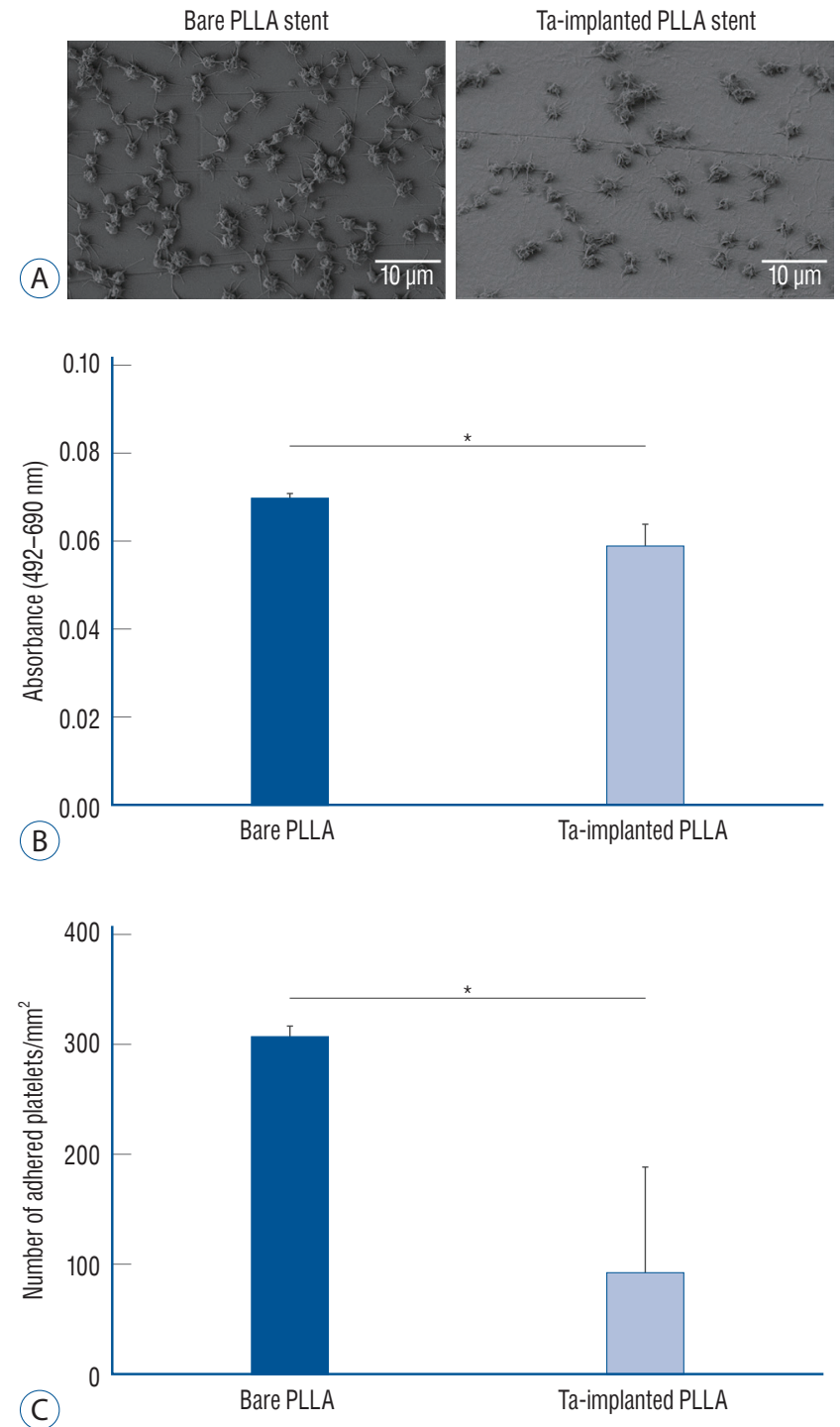

Fig. 9. Platelet adhesion test. A : Scanning electron microscope images of the adhered platelet morphology on bare and tantalum-implanted poly-L-lactic acid (PLLA) stents. B : Absorbance difference between 492 $\mathrm{nm}$ and $690 \mathrm{~nm}$ of a platelet-lysed solution and (C) the number of adhered platelets on each PLLA stent from the lactate dehydrogenase assay. ${ }^{*} p<0.05$. 
$104.1^{\circ} \pm 0.44^{\circ}$ on the bare PLLA ( $\left.p=0.000\right)$; that is, the Ta treatment increased the hydrophilicity of the stent by introducing a Ta oxide layer. As depicted in Fig. 6, the Ta-implantation
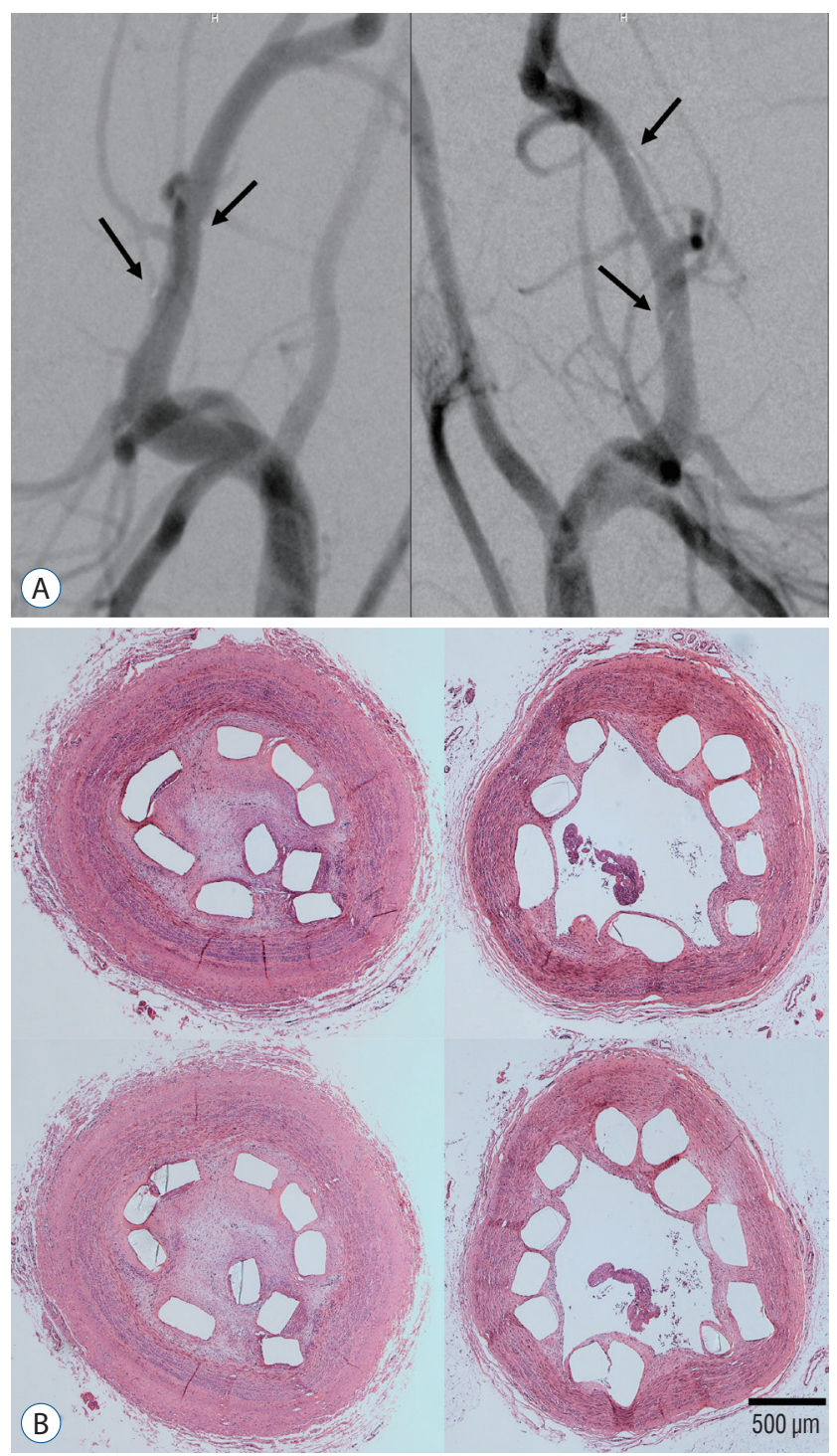

Fig. 10. Angiographic and histologic results ( $\times 40$ magnification) 3 months after stent deployment. A : All parent arteries are patent in the angiographic images without in-stent stenosis (right: tantalum-implanted poly-L-lactic acid [PLLA] stent, left : bare PLLA stent). The black arrows indicate the gold marker of the stent. B : After tissue preparation and hematoxylin and eosin staining with identical specimens, many specimens demonstrate what appears to be an arterial lumen collapse despite the lack of evidence of stenosis in the angiographic images. The discrepancy between the angiographic and histological results may be due to issues that arose during the tissue preparation step with paraffin embedding for the immunohistochemical analysis and stent characteristics such as the small diameter and weak radial force (right : tantalum-implanted PLLA stent, left : bare PLLA stent). process on the surface did not affect the mechanical strength of the PLLA stent, indicating that good mechanical integrity remained.

Fig. 7 shows that Ta ions were successfully implanted into both the luminal and abluminal surfaces of the PLLA stent, forming a Ta-implanted skin layer regardless of the location. Because the distance from the Ta target and physical hindrance could affect the efficiency of Ta implantation on the luminal surface, the atomic concentration of Ta was higher on the abluminal surface. However, even on the luminal surface, the Ta layer was clearly observed at a depth of $10 \mathrm{~nm}$ on the topmost surface with the highest atomic concentration of approximately $6 \%$.

\section{In vitro endothelialization and blood compatibility}

The effects of Ta ion implantation on the interaction between the HUVECs, platelets and the PLLA stent surface were examined by visualizing the corresponding adhesion morphologies on bare and Ta-implanted PLLA substrates. Fig. 8A shows representative CLSM images of the adhered HUVECs after 1, 4, and 7 days of culturing. On the Ta-implanted PLLA surface, a significant number of HUVECs were adhered and progressively spread in every direction, forming a typical cobblestone-like morphology with tight cell-cell interaction, whereas the bare PLLA exhibited fewer cells which were adhered individually with a lack of cell-cell junctions. The fractions of cell surface coverage with culturing times of 1,4 , and 7 days were $48 \%, 61 \%$, and $77 \%$ on the Ta-implanted PLLA, respectively, and were $14 \%, 34 \%$, and $40 \%$ on the bare PLLA

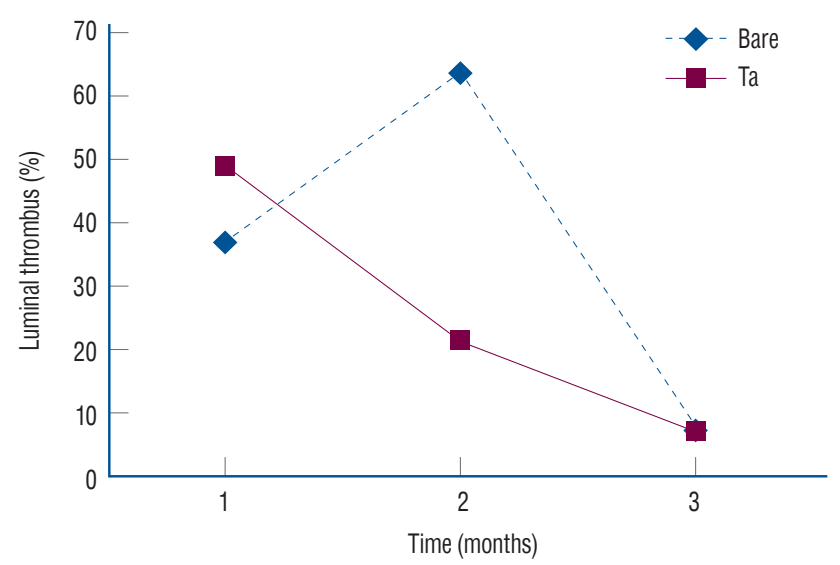

Fig. 11. In vivo thrombogenicity. The tantalum (Ta)-implanted poly-Llactic acid stent group shows less thrombus formation according to the 2-month follow-up exam (21.2\% vs. 63.9\%, $p=0.005)$. 
(Fig. 8B). In addition, HUVECs tended to proliferate more actively on the Ta-implanted PLLA, showing a higher cell viability value when compared to those cultured on bare PLLA (Fig. 8C).

The blood compatibility of each sample was investigated by platelet adhesion tests and the results were compared quantitatively. As shown in Fig. 9A, significantly fewer platelets were
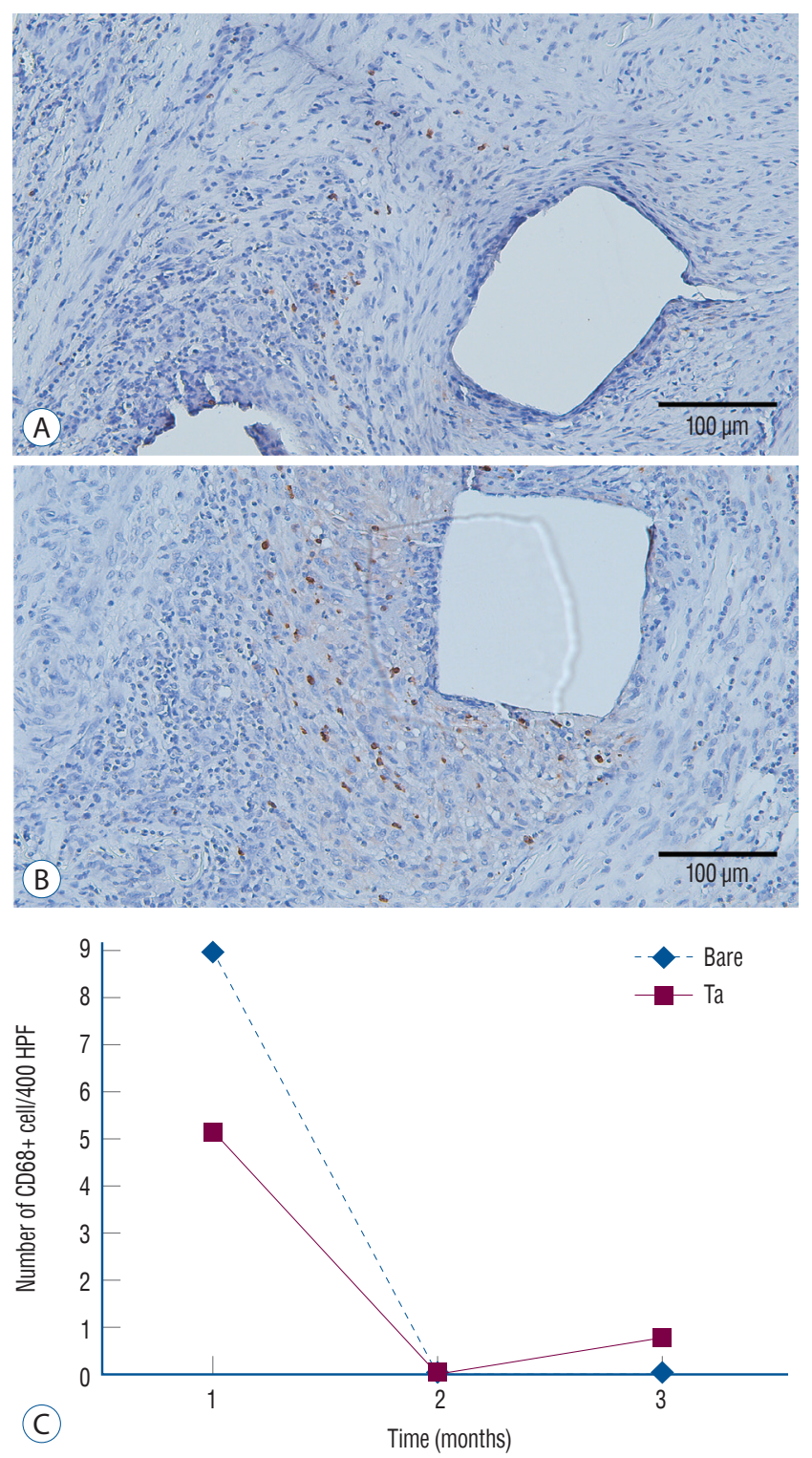

Fig. 12. In vivo inflammation. Less of an inflammatory reaction is identified in the tantalum (Ta)-implanted poly-L-lactic acid (PLLA) stent group at 1-month follow-up. The numbers of CD68+ cells/400 HPF are $5.2 \pm 7.3$ in the Ta-implanted PLLA stent group (A) and 9.0 \pm 16.2 in the bare PLLA stent group (B) ( $\times 200$ magnification). However, these outcomes lack statistical significance $(p=0.601) . C$ : The inflammatory response decreases with time. found to have adhered when the PLLA surface was treated with Ta ion implantation; the density of the adhered platelets (number/ $\mathrm{mm}^{2}$ ) on the Ta-implanted PLLA was $92.2 \pm 95.5$, significantly lower than that on the bare PLLA $(306.0 \pm 10.2$, $p=0.046)$ (Fig. 9B and C).

\section{In vivo test}

The follow-up angiographic images showed that all ECAs were patent with no evidence of in-stent stenosis (Fig. 10A). However, an arterial lumen collapse was identified in the histologic analysis (Fig. 10B). Therefore, an accurate analysis of percent area stenosis ([1 - luminal area/internal elastic lamina] $\times 100$ ) based on a histologic examination could not be done. Luminal thrombus formation was significantly suppressed in the Ta-implanted PLLA stent group at 2 months after stent placement (21.2\% vs. $63.9 \%, p=0.005$, Fig. 11). Neointimal hyperplasia, luminal stenosis, inflammation and fibrosis showed no differences between the two groups.

The expression levels of CD 31, CD 34, and anti-smooth muscle cell alpha actin showed no significant differences between the two groups in the immunohistochemical analysis. CD 68(+) cells, which represent an inflammatory response, were more frequently identified around the bare PLLA stent during the 1-month follow-up (Fig. 12A and B), and the difference disappeared over time (Fig. 12C).

\section{DISCUSSION}

Our study aimed to investigate the biological effects of Ta ion implantation on the re-endothelialization and thrombogenicity of PLLA stents to assess whether this surface treatment technique can ameliorate the serious complications associated with bare PLLA stents. Although PLLA stents for cardiovascular applications have been approved by the Food and Drug Administration in the USA and have been used in clinical practice ${ }^{24}$, they are currently being withdrawn from the market due to excessive inflammation and thrombus formation ${ }^{4,11,16,20)}$. Because these problems are caused by hypersensitive reactions to the PLLA surface in the vessel, biocompatible coatings have been demonstrated as an effective and suitable treatment capable of reducing the risk ${ }^{22}$. In particular, previous studies have revealed that the surface of Ta is hydrophilic and biologically stable, enabling favorable interaction 
between endothelial cells and blood proteins ${ }^{1,13}$.

Ta is a well-known biocompatible metal used in various medical applications, of which oxides enhance its hemocompatibility by preventing the charge transfer of fibrinogen in the blood, resulting in the restricted formation of thrombo$\mathrm{sis}^{3)}$. It was reported relatively few metal ions rarely cause cytotoxicity, allergies, or other biological side-effects ${ }^{5}$. Thus, Ta implantation is able to apply on a biodegradable intravascular stent because the fine Ta or its oxide particles could be released out and partially dissolved and transported along the normal circulation.

In this study, we confirmed that the surfaces of the PLLA stents were successfully modified with Ta ion implantation. Sputtered Ta ions from a target gun were implanted into the topmost surface of the PLLA stent, forming a Ta-implanted skin layer with a thickness of around $10 \mathrm{~nm}$ on its surface (Fig. 7). After the Ta ion implantation treatment, the PLLA stent maintained its original 3D geometry and surface flatness without any micro-scale unevenness or defects (Fig. 3), which should minimize turbulent intraluminal blood flows and provide a stable environment at the implantation site ${ }^{23}$.

In vitro studies showed that Ta ion implantation substantially improved the endothelialization ability of the PLLA stent, whereas platelet attachment was suppressed on its surface. These favorable interactions of the Ta-implanted PLLA stent with cells can enhance the in vivo biocompatibility.

Prior to a discussion with histological results, we would like to highlight a practical difficulty which arises during the preparation of histologic specimens. In general, resin embedding is used in intravascular stent experiments to maintain the integrity of the stent structure. However, in this study, relatively weak paraffin embedding was selected in most specimen preparation cases for the immunohistochemical analysis. In addition, the relatively small size of the PLLA stent $(3 \times 10$ $\mathrm{mm}$ ) complicated the maintenance of the original shape during the histological sample preparation process. Despite the fact that the histologic examination showed what appeared to be an arterial occlusion in a segment of the stent, the angiographic results clearly indicated that both stents were widely patent without any signs of in-stent stenosis. Therefore, it should be reasonable to conclude that the angiographic results are more reliable with respect to the long-term patency rate of the stent.

Anti-thrombogenicity is a crucial characteristic of a hydro- phobic PLLA polymer stent, as it allows the suppression of platelet aggregation and prevents in-stent restenosis or late thrombosis, leading possibly to clinical failures. Although there were no differences found during the 1-month follow-up assessment, less thrombogenicity was identified at the followup exam after 2 months in a histologic analysis. Interpretation of this result is described below.

A critical limitation of the in vivo test appears to be the use of a balloon to deploy the PLLA stent in the artery. We used coronary balloons with a diameter of $4 \mathrm{~mm}$, greater by $1 \mathrm{~mm}$ than the stent diameter used to reduce the recoil phenomenon of PLLA stents caused by the mechanical properties of PLLA. The diameter of the ECA was approximately $3 \mathrm{~mm}$; therefore, balloon inflation for the deployment of the PLLA stent may damage the arterial wall. As a result, the evaluation of thrombosis may have been affected by arterial damage during an acute period. However, over time, the arterial wall would have healed. The result of thrombogenicity in the 2-month followup assessment appears to stem from the healing of the damaged arterial wall. Under such conditions, the Ta-implanted PLLA stent showed less thrombogenicity, in good agreement with the results of the in vitro experiment.

Rapid endothelialization after stent deployment is essential to prevent intimal hyperplasia or restenosis. In the evaluation of endothelialization (CD 31, CD 34) and the tissue response (anti-smooth muscle cell alpha actin), there were no differences between the two stent groups. It appears that the effect of arterial damage due to the inflation of the balloon is more prevalent than that of the PLLA stent itself. In addition, the longest follow-up duration after stent deployment was only 3 months in this study, which may be too short a period of time to assess endothelialization. The results of CD 68-positive cells, which represent inflammation, also appear to be strongly influenced by balloon-induced arterial wall damage. If we can increase the number of experimental animals for an acute-phase evaluation and minimize damage to blood vessels as caused by the balloons, more significant results can be gained.

This preclinical study of Ta-implanted PLLA stents presents some implications for future research on biodegradable PLLA stents in the cerebrovascular field. In a swine model, the balloon-mounted PLLA stent, which has a similar navigability compared to a conventional balloon-mounted coronary stent, was easily navigated to the ECA with a 6 Fr guiding catheter. 
Of course, the arterial anatomy of the swine was straight, and the navigability of the stent was not an issue. More flexibility may be needed for application to the cerebral arteries to overcome the tortuosity of the internal carotid artery. Further research is therefore needed to improve the navigability of the stent.

The use of balloons may not be suitable, especially for the treatment of an intracranial aneurysm patient owing to the high risk of arterial damage during the deployment of the stent. Therefore, it is necessary to create a type of self-expandable stent to minimize intraoperative vascular injuries. In order to make a biocompatible and self-expandable stent with PLLA, it is necessary to modify not only the surface biological properties but also the bulk mechanical strength of the PLLA, including the elastic modulus, toughness, and strain-to-failure levels. In previous studies, blending with several biopolymers such as cellulose, abaca fibers, chitosan, polyvinyl acetate, and low-density polyethylene has shown the ability to enhance the mechanical properties of PLLA ${ }^{2,17,21)}$. We expect that a better understanding of the biological performance capabilities of Ta-implanted PLLA stents will be achieved by developing these devices in a self-expandable form, followed by testing in an in vivo model in the future.

\section{CONCLUSION}

A novel strategy for developing a more biocompatible and less thrombogenic PLLA stent has been demonstrated. The Ta ion implantation technique was able to produce a Ta-implanted surface layer without any deformation or distortion of the PLLA stent. The biological properties of the PLLA stent became remarkably more favorable by enhancing endothelial cell responses and suppressing platelet adhesion. The swine in vivo study conducted here confirmed the lower level of thrombogenicity of the Ta-implanted PLLA stent as compared to that with a bare stent.

\section{CONFLICTS OF INTEREST}

No potential conflict of interest relevant to this article was reported.

\section{INFORMED CONSENT}

This type of study does not require informed consent.

\section{AUTHOR CONTRIBUTIONS}

\author{
Conceptualization : WSC, TSJ, HSK, JEK, HEK \\ Data curation : KK, SP, SML \\ Formal analysis : KK, SP, JHP, SML \\ Funding acquisition : WSC, TSJ, HSK, JEK, HEK \\ Methodology : KK, SP, SML, TSJ, HSK, HEK \\ Project administration : KK, SP, TSJ, HSK, HEK \\ Visualization : KK, SP, TSJ, HSK, HEK \\ Writing - original draft : KK, SP \\ Writing - review \& editing : HSK, TSJ, HEK
}

\section{ORCID}

$\begin{array}{ll}\text { Kangmin Kim } & \text { https://orcid.org/0000-0002-7409-4412 } \\ \text { Suhyung Park } & \text { https://orcid.org/0000-0002-1808-018X } \\ \text { Jeong Hwan Park } & \text { https://orcid.org/0000-0003-4522-9928 } \\ \text { Won-Sang Cho } & \text { https://orcid.org/0000-0002-3345-8718 } \\ \text { Hyoun-Ee Kim } & \text { https://orcid.org/0000-0001-8542-0124 } \\ \text { Sung-Mi Lee } & \text { https://orcid.org/0000-0002-0130-1572 } \\ \text { Jeong Eun Kim } & \text { https://orcid.org/0000-0002-6927-3109 } \\ \text { Hyun-Seung Kang https://orcid.org/0000-0002-6957-1907 } \\ \text { Tae-Sik Jang } & \text { https://orcid.org/0000-0003-3540-0525 }\end{array}$

\section{- Acknowledgements}

This work was funded by the Interdisciplinary Research Initiatives Program by College of Engineering and College of Medicine, Seoul National University (2017).

\section{References}

1. Barbarash LS, Bolbasov EN, Antonova LV, Matveeva VG, Velikanova EA, Shesterikov EV, et al. : Surface modification of poly-e-caprolactone electrospun fibrous scaffolds using plasma discharge with sputter deposition of a titanium target. Materials Letters $171:$ 87-90, 2016

2. Bledzki AK, Jaszkiewicz A, Scherzer D : Mechanical properties of PLA composites with man-made cellulose and abaca fibres. Compos Part 
A Appl Sci Manuf 40 : 404-412, 2009

3. Chen JY, Leng YX, Tian XB, Wang LP, Huang N, Chu PK, et al. : Antithrombogenic investigation of surface energy and optical bandgap and hemocompatibility mechanism of $\mathrm{Ti}\left(\mathrm{Ta}^{+5}\right) \mathrm{O}_{2}$ thin films. Biomaterials $23:$ 2545-2552, 2002

4. Collet C, Asano T, Miyazaki Y, Tenekecioglu E, Katagiri Y, Sotomi Y, et al. : Late thrombotic events after bioresorbable scaffold implantation: a systematic review and meta-analysis of randomized clinical trials. Eur Heart J 38 : 2559-2566, 2017

5. Hanawa $\mathrm{T}$ : Metal ion release from metal implants. Mat Sci Eng C-Bio S 24 : 745-752, 2004

6. Hou LD, Li Z, Pan Y, Sabir M, Zheng YF, Li L : A review on biodegradable materials for cardiovascular stent application. Front Mater Sci 10 : 238-259, 2016

7. Hu T, Yang C, Lin S, Yu Q, Wang G : Biodegradable stents for coronary artery disease treatment: recent advances and future perspectives. Mater Sci Eng C Mater Biol Appl 91 : 163-178, 2018

8. Hwang G, Kim JG, Song KS, Lee YJ, Villavicencio JB, Suroto NS, et al. : Delayed ischemic stroke after stent-assisted coil placement in cerebral aneurysm: characteristics and optimal duration of preventative dual antiplatelet therapy. Radiology 273 : 194-201, 2014

9. Ikarashi Y, Toyoda K, Ohsawa N, Uchima T, Tsuchiya T, Kaniwa M, et al. : Comparative studies by cell culture and in vivo implantation test on the toxicity of natural rubber latex materials. J Biomed Mater Res 26 : 339-356, 1992

10. Jin W, Wang G, Lin Z, Feng H, Li W, Peng $X$, et al. : Corrosion resistance and cytocompatibility of tantalum-surface-functionalized biomedical ZK60 Mg alloy. Corros Sci 114 : 45-56, 2017

11. Kereiakes DJ, Ellis SG, Metzger C, Caputo RP, Rizik DG, Teirstein PS, et al. : 3-year clinical outcomes with everolimus-eluting bioresorbable coronary scaffolds: the ABSORB III trial. J Am Coll Cardiol 70 : 28522862, 2017

12. Krischek Ö, Miloslavski E, Fischer S, Shrivastava S, Henkes H : A comparison of functional and physical properties of self-expanding intracranial stents [Neuroform3, Wingspan, Solitaire, Leo(+), Enterprise]. Minim Invasive Surg $54: 21-28,2011$

13. Nie FL, Zheng YF, Wang Y, Wang JT : Microstructures, mechanical behavior, cellular response, and hemocompatibility of bulk ultrafinegrained pure tantalum. J Biomed Mater Res B Appl Biomater 102 :
221-230, 2014

14. Park C, Seong YJ, Kang IG, Song EH, Lee H, Kim J, et al. : Enhanced osseointegration ability of poly(lactic acid) via tantalum sputtering-based plasma immersion ion implantation. ACS Appl Mater Interfaces 11 : 10492-10504, 2019

15. Pizzoferrato A, Ciapetti G, Savarino L, Stea S, Tarabusi C : Results of histological grading on 100 cases of hip prosthesis failure. Biomaterials 9: 314-318, 1988

16. Räber L, Brugaletta S, Yamaji K, O'Sullivan CJ, Otsuki S, Koppara T, et al. : Very late scaffold thrombosis: intracoronary imaging and histopathological and spectroscopic findings. J Am Coll Cardiol 66 : 1901-1914, 2015

17. Rasal RM, Janorkar AV, Hirt DE : Poly(lactic acid) modifications. Prog Polym Sci 35 : 338-356, 2010

18. Schwartz RS, Huber KC, Murphy JG, Edwards WD, Camrud AR, Vlietstra $R E$, et al. : Restenosis and the proportional neointimal response to coronary artery injury: results in a porcine model. J Am Coll Cardiol 19 : 267-274, 1992

19. Sharkawi T, Cornhill F, Lafont A, Sabaria P, Vert M : Intravascular bioresorbable polymeric stents: a potential alternative to current drug eluting metal stents. J Pharm Sci 96 : 2829-2837, 2007

20. Stone GW, Ellis SG, Gori T, Metzger DC, Stein B, Erickson M, et al. : Blinded outcomes and angina assessment of coronary bioresorbable scaffolds: 30-day and 1-year results from the ABSORB IV randomised trial. Lancet 392 : 1530-1540, 2018

21. Suyatma NE, Copinet A, Tighzert $L$, Coma V : Mechanical and barrier properties of biodegradable films made from chitosan and poly (lactic acid) blends. J Polym Environ 12 : 1-6, 2004

22. Tverdokhlebov SI, Bolbasov EN, Shesterikov EV, Antonova LV, Golovkin AS, Matveeva VG, et al. : Modification of polylactic acid surface using RF plasma discharge with sputter deposition of a hydroxyapatite target for increased biocompatibility. Appl Surf Sci 329 : 32-39, 2015

23. Wang J, Jin $X$, Huang $Y$, Ran $X$, Luo D, Yang D, et al. : Endovascular stent-induced alterations in host artery mechanical environments and their roles in stent restenosis and late thrombosis. Regen Biomater 5 : 177-187, 2018

24. Wiebe J, Nef HM, Hamm CW : Current status of bioresorbable scaffolds in the treatment of coronary artery disease. J Am Coll Cardiol 64 : 2541-2551, 2014 